CORRIGENDUM

doi:10.1038/nature12933

\title{
Corrigendum: Primary forests are irreplaceable for sustaining tropical biodiversity
}

Luke Gibson, Tien Ming Lee, Lian Pin Koh, Barry W. Brook, Toby A. Gardner, Jos Barlow, Carlos A. Peres, Corey J. A. Bradshaw, William F. Laurance, Thomas E. Lovejoy \& Navjot S. Sodhi

Nature 478, 378-381 (2011); doi:10.1038/nature10425

We identified a conversion error in some of the biodiversity values used in our meta-analysis. The meta-analysis requires both mean and standard deviation values for each record, and several studies reported 95\% confidence intervals instead of standard deviation. We incorrectly converted from these confidence intervals to estimate standard deviation, affecting 164 rows $(7.4 \%)$ of the 2,220 rows in our database. Instead of dividing $\sqrt{n} \times$ (upper confidence interval minus mean) by 1.96 , we multiplied by 1.96 . Because both primary forest and disturbed forest categories were affected, there was no bias towards or against any category. After correcting for this conversion error, our results did not alter substantially (see Table 1 of this Corrigendum). All statements in our Letter remain fully supported, and most effect sizes increased, further strengthening our conclusions. N.S.S. is deceased.

Table 1 | Corrected effect sizes from Supplementary Table 1

\begin{tabular}{|c|c|c|c|}
\hline & & $\begin{array}{l}\text { Original effect } \\
\text { size }\end{array}$ & $\begin{array}{l}\text { Corrected effect } \\
\text { size }\end{array}$ \\
\hline Overall & & 0.51 & 0.56 \\
\hline \multirow[t]{4}{*}{ Continent } & Africa & 0.34 & 0.46 \\
\hline & Asia & 0.95 & 1.07 \\
\hline & Central America & 0.10 & 0.09 \\
\hline & South America & 0.44 & 0.46 \\
\hline \multirow[t]{4}{*}{ Taxon } & Arthropods & 0.64 & 0.69 \\
\hline & Birds & 0.72 & 0.81 \\
\hline & Mammals & -0.12 & -0.10 \\
\hline & Plants & 0.58 & 0.64 \\
\hline \multirow[t]{5}{*}{ Metric } & Abundance & 0.19 & 0.20 \\
\hline & $\begin{array}{l}\text { Community structure } \\
\text { and function }\end{array}$ & 0.41 & 0.41 \\
\hline & Demographics & 0.00 & -0.01 \\
\hline & Forest structure & 0.75 & 0.75 \\
\hline & Richness & 0.83 & 0.98 \\
\hline \multirow[t]{12}{*}{ Disturbance } & Abandoned agriculture & 1.05 & 1.05 \\
\hline & Agriculture & 1.04 & 1.24 \\
\hline & Agroforestry & 0.65 & 0.76 \\
\hline & Burned & 0.87 & 0.90 \\
\hline & Clear-cut & 2.31 & 2.31 \\
\hline & Hunted and disturbed & 0.01 & 0.01 \\
\hline & Other extraction & 0.59 & 0.59 \\
\hline & Pastures & 0.48 & 0.49 \\
\hline & Plantations & 0.50 & 0.65 \\
\hline & Secondary & 0.41 & 0.45 \\
\hline & Selectively logged & 0.11 & 0.12 \\
\hline & Shaded plantations & 0.65 & 0.65 \\
\hline
\end{tabular}

